\title{
Determination of fracture parameters for a bi-material center cracked plate subjected to biaxial loading using FEOD method
}

\begin{abstract}
Atefeh Mousavi ${ }^{a^{*}}$ and M.R.M. Aliha ${ }^{b}$
a Department of Biomedical Engineering, Science and Research Branch, Islamic Azad University, Tehran, Iran

${ }^{b}$ Welding and Joining Research Center, School of Industrial Engineering, Iran University of Science and Technology (IUST), Narmak, 16846-13114, Tehran, Iran

\begin{tabular}{l}
\hline A R T I C L EI N F O \\
\hline Article history: \\
Received 6 October, 2015 \\
Accepted 27 February 2016 \\
Available online \\
27 February 2016 \\
\hline Keywords: \\
bi-material center crack plate \\
stress field \\
FEOD \\
fracture parameters
\end{tabular}

A B S T R A C T

Fracture parameters of a bi-material plate containing a cener crack and subjected to biaxial tensile loading was calculated numerically. Based on the crack tip stress field obtained numerically in a bi-material joint and using the finite element over deterministic (FEOD) method, the stress intensity factors $\left(K_{\mathrm{I}}\right.$ and $\left.K_{\mathrm{II}}\right)$ and also non-singular $T$-stress terms, were determined for different material properties and biaxial loading cases. Due to asymmetry of loading and material properties in the investigated dissimilar plate, the center crack experinces mixed mode I/II fracture in general. By increasing the bi-material constant value, which shows the difference between the mechanical properties of two materials, the amplitude of stress intensity factor decreases. The obtained results from this method were in good agreement with the displacment field method prevousiuly reported by other researchers.
\end{abstract}

C 2016 Growing Science Ltd. All rights reserved.

\section{Introduction}

Fracture growth from the tip of cracks in the interface of bi-material components is one of the major failure modes in such materials. Bi-marerial joints are frequently used in various engineering structures such as dentin/restorative (Ensaff et al., 2001; Toparli \& Aksoy, 1998), bone/cement (Wang \& Agrawal, 2000) ceramic/ceramic (Mirsayar and Park, 2016), ceramic/metal (Hutchinson \& Suo, 1992; Mirsayar, 2013; Mirsayar \& Samaei 2014), rack/concrete (Zhong et al., 2011), asphalt concret/overley or bridge deck (Hakimzadeh et al., 2012) and etc. Due to some technical problems such as lack of suitable bonding, a crack may initiate in the interface of bi-matrial and then can propagate during service life of such joints because of applied loads and external or internal stresses. In practice, bimatrial cracked components experince mixed mode I/II deformations under in-plane loads due to

* Corresponding author.

E-mail addresses: mrm aliha@iust.ac.ir (M.R.M. Aliha) 
complex type of loading and also asymmetry of material at the interface. Such cracks may grow along the interface in the weak joints or kink into one of the materials in strong interfaces. In order to study the onest of interface fracture based on the approach of fracture mechanics, the stress/strain field at the tip of crack should be known. Some researchers have investigated the interface fracture problem using eaither crack tip stress or displacement fields. Williams (1959), Rice and Sih (1965), Asaro et al. (1993), Ravichandran and Ramesh (2005), Morioka and Sun (2010), Mirsayar (2014), Mirsayar et al. (2014) are to name afew. For example, Williams (1959) outlined the stress field for the general bi-material interface problem. The elastic stress field around a bi-matrial crack tip can be written as following equation in the cartesian coordinates:

$$
\begin{aligned}
& {\left[\begin{array}{c}
\sigma_{x}^{m} \\
\sigma_{y}^{m} \\
\tau_{x y}^{m}
\end{array}\right]=} \\
& \sum_{N=0,2,4, \ldots,}^{N} k_{I N} r^{\frac{(n-1)}{2}}\left\{\begin{array}{l}
S^{m}\left\{3 \cos \left[\frac{N-1}{2} \theta-\varepsilon \ln \left(\frac{r}{L}\right)\right]-(N-1) \sin \theta \sin \left[\frac{N-3}{2} \theta-\varepsilon \ln \left(\frac{r}{L}\right)\right]+2 \varepsilon \sin \theta \cos \left[\frac{N-3}{2} \theta-\varepsilon \ln \left(\frac{r}{L}\right)\right]\right\}-\frac{1}{S^{m}} \cos \left[\frac{N-1}{2} \theta+\varepsilon \ln \left(\frac{r}{L}\right)\right] \\
S^{m}\left\{\cos \left[\frac{N-1}{2} \theta-\varepsilon \ln \left(\frac{r}{L}\right)\right]+(N-1) \sin \theta \sin \left[\frac{N-3}{2} \theta-\varepsilon \ln \left(\frac{r}{L}\right)\right]-2 \varepsilon \sin \theta \cos \left[\frac{N-3}{2} \theta-\varepsilon \ln \left(\frac{r}{L}\right)\right]\right\}+\frac{1}{S^{m}} \cos \left[\frac{N-1}{2} \theta+\varepsilon \ln \left(\frac{r}{L}\right)\right] \\
S^{m}\left\{-\sin \left[\frac{N-1}{2} \theta-\varepsilon \ln \left(\frac{r}{L}\right)\right]-(N-1) \sin \theta \cos \left[\frac{N-3}{2} \theta-\varepsilon \ln \left(\frac{r}{L}\right)\right]-2 \varepsilon \sin \theta \sin \left[\frac{N-3}{2} \theta-\varepsilon \ln \left(\frac{r}{L}\right)\right]\right\}+\frac{1}{S^{m}} \sin \left[\frac{N-1}{2} \theta+\varepsilon \ln \left(\frac{r}{L}\right)\right]
\end{array}\right\}+ \\
& \sum_{M=1,3,5, \ldots, \frac{k_{I I M}(m)}{R^{(m)}} r^{\frac{(M-1)}{2}}}\left\{\begin{array}{c}
4 \cos \left[\frac{M-1}{2} \theta\right]-(M-1) \sin \theta \sin \left[\frac{M-3}{2} \theta\right] \\
(M-1) \sin \theta \sin \left[\frac{M-3}{2} \theta\right] \\
-2 \sin \left[\frac{M-1}{2} \theta\right]-(M-1) \sin \theta \cos \left[\frac{M-3}{2} \theta\right]
\end{array}\right\}+ \\
& \Sigma_{N=0,2,4, \ldots, \frac{k_{I N}}{Q}}^{N} r^{\frac{N-1)}{2}}\left\{\begin{array}{l}
S^{m}\left\{3 \sin \left[\frac{N-1}{2} \theta-\varepsilon \ln \left(\frac{r}{L}\right)\right]+(N-1) \sin \theta \cos \left[\frac{N-3}{2} \theta-\varepsilon \ln \left(\frac{r}{L}\right)\right]+2 \varepsilon \sin \theta \sin \left[\frac{N-3}{2} \theta-\varepsilon \ln \left(\frac{r}{L}\right)\right]\right\}+\frac{1}{S^{m}} \sin \left[\frac{N-1}{2} \theta+\varepsilon \ln \left(\frac{r}{L}\right)\right] \\
S^{m}\left\{\sin \left[\frac{N-1}{2} \theta-\varepsilon \ln \left(\frac{r}{L}\right)\right]-(N-1) \sin \theta \cos \left[\frac{N-3}{2} \theta-\varepsilon \ln \left(\frac{r}{L}\right)\right]-2 \varepsilon \sin \theta \cos \left[\frac{N-3}{2} \theta-\varepsilon \ln \left(\frac{r}{L}\right)\right]\right\}-\frac{1}{S^{m}} \sin \left[\frac{N-1}{2} \theta+\varepsilon \ln \left(\frac{r}{L}\right)\right] \\
S^{m}\left\{\cos \left[\frac{N-1}{2} \theta-\varepsilon \ln \left(\frac{r}{L}\right)\right]-(N-1) \sin \theta \sin \left[\frac{N-3}{2} \theta-\varepsilon \ln \left(\frac{r}{L}\right)\right]+2 \varepsilon \sin \theta \cos \left[\frac{N-3}{2} \theta-\varepsilon \ln \left(\frac{r}{L}\right)\right]\right\}+\frac{1}{S^{1}} \cos \left[\frac{N-1}{2} \theta+\varepsilon \ln \left(\frac{r}{L}\right)\right]
\end{array}\right\}+ \\
& \sum_{M=1,3,5, \ldots \ldots \frac{k_{I I M}}{R(M)}} r^{\frac{(M-1)}{2}}\left\{\begin{array}{c}
2 \sin \left[\frac{M-1}{2} \theta\right]+(M-1) \sin \theta \sin \left[\frac{M-3}{2} \theta\right] \\
2 \sin \left[\frac{M-1}{2} \theta\right]-(M-1) \sin \theta \cos \left[\frac{M-3}{2} \theta\right] \\
-(M-1) \sin \theta \sin \left[\frac{M-3}{2} \theta\right]
\end{array}\right\}
\end{aligned}
$$

where, $N$ and $M$, are the terms of mode $I$ and mode $I I$ parameters in the William's series expansion, respectively the constants of singular terms in Eq. (1) are known as the modes $I$ and $I I$ stress intensity factors $\left(K_{\mathrm{I}}\right.$ and $\left.K_{\mathrm{II}}\right)$. The coefficient of the second term in this equation (which is non-singular stress term) is called $T$-stress. $r$ and $\theta$ are polar coordinates as defined in Fig. 1 for a biomaterial crack tip and $L$ is a characteristic length. In comparison with the crack tip stress field in a homogenous medium (which its stress singularity is in the form of $r^{-1 / 2}$, the character of singularity changes to $r^{-1 / 2+i \varepsilon}$ for bimaterials, in which $\varepsilon$ is the bi-material constant defined as :

$$
\varepsilon=\frac{1}{2 \pi}\left(\frac{G_{2} K_{1}+G_{1}}{G_{1} K_{2}+G_{2}}\right)
$$

where $G_{\mathrm{m}}$ is the shear modulus, $\kappa_{m}=\left(3-v_{m}\right) /\left(1+v_{m}\right)$ for plane stress and 3-4 $v_{m}$ for plane strain. Also in Eqs. 1 and 2, $\mathrm{m}=(1,2)$ denotes material number of bi-material joint. Other constants used for defining material-1, in Eq. 1 are: $=2 \sqrt{2 \pi} \cosh (\pi \varepsilon), S^{(1)}=\mathrm{e}^{-\varepsilon(\pi-\beta)}, R^{(1)}=\sqrt{2 \pi}(1+\omega), \omega=$ $\frac{\left(1+k_{1}\right) G_{2}}{\left(1+k_{2}\right) G_{1}}$. The stress field expressions for material-2 can be obtained by replacing $\mathrm{e}^{-\varepsilon \pi}$ by $\mathrm{e}^{+\varepsilon \pi}, \mathrm{e}^{+\varepsilon \pi}$ by $\mathrm{e}^{-}$ $\varepsilon \pi, \omega$ by $1 / \omega$ and replacing the corresponding $\mathrm{R}^{(2}$ ) and $\mathrm{S}^{(2)}$ into the Eq. 1 (Ravichandran and Ramesh 2005). 


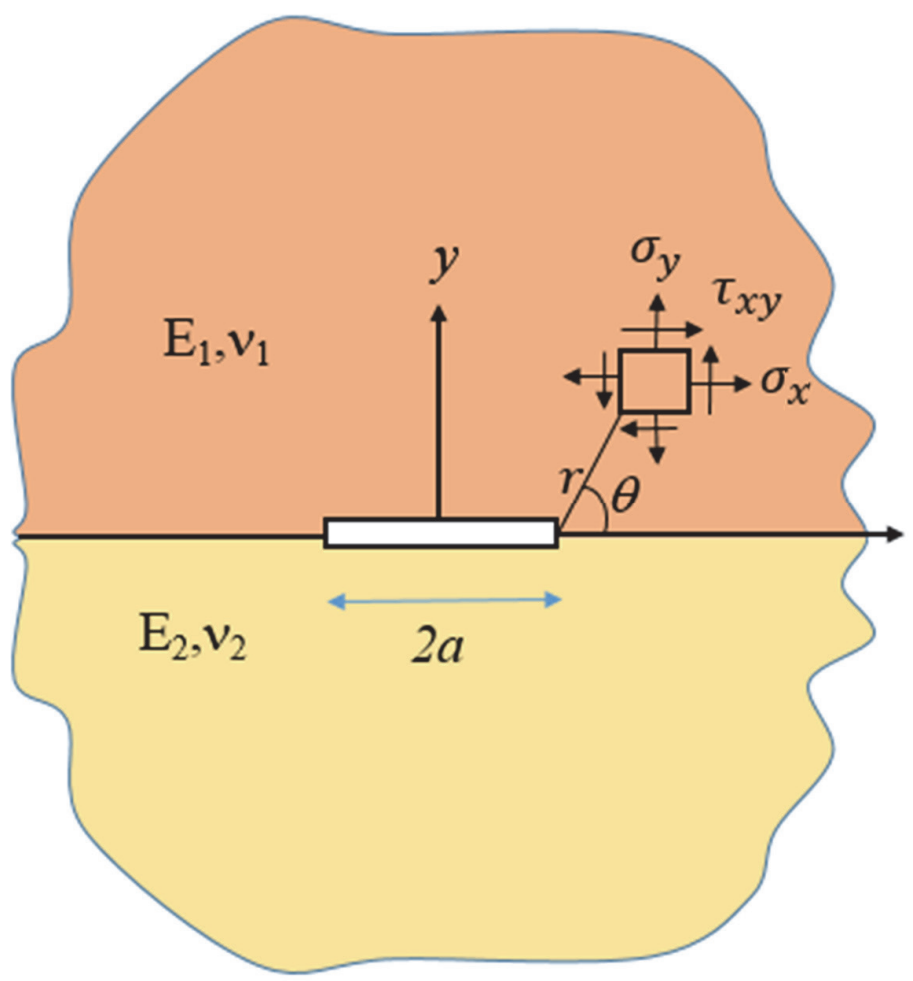

Fig. 1. Stress field in the vicinity of interface crack initiated in a bi-material medium

Three fracture parameters $\left(K_{\mathrm{I}}, K_{\mathrm{II}}\right.$ and $\left.T\right)$ that define the crack tip stress field for a bi-material joint can then be used for evaluating the onset of interface fracture via the available fracture theories such as (Mirsayar, 2014). Therefore, it is necessary to determine these parameters for any given interface crack problem with any arbitrary geometry, material type and loading conditions. Since there is no general analytical solution for determining these fracture parameters in bi-material joints, the use of numerical methods are powerful tools for computing them. The commercial finite element codes can be successfully used for computing the fracture parameters (Abd-Elhady, 2013; Ayatollahi \& Aliha, 2007; 2009, Ayatollahi et al., 2011; Aliha et al., 203, 2015). However, for bi-material interface fracture problems, the available finite element codes may not provide these parameters directly and with good accuracy. Hence in this paper, as a typical bi-material interface problem, the fracture parameters of a bi-material center crack plate subjected to different biaxial loading conditions are computed numerically from its crack tip stress field and by employing a finite element over deterministic (FEOD) method. It is shown that the stress based FEOD method can provide accurate results for the fracture parameters of the bi-material center cracked plate.

\section{Finite element over deterministic (FEOD) method}

The over-deterministic method makes use of a large number of data points to determine a small set of unknown coefficients from a large system of equations (Ayatollahi \& Nejati 2011). For example and as a practical application, the FEOD method can be used for determining a selected number of coefficients in the William's series expansion form the stress components which can be obtained from finite element analysis. In order to use the FEOD method for a bi-material crack medium, the following relation between the stress components and constants of the William's series expansion can be written:

$$
[\sigma]_{2 k \times 1}=[C]_{2 k \times(N+M+2)}[X]_{(N+M+2) \times 1}
$$


where $\sigma$ is the components of stress tensor, $\mathrm{C}$ is a matrix that its components are functions of $r$ and $\theta$, and $\mathrm{X}$ is the matrix of unknown variables (i.e. the Williams constants). By selecting a large number of nodes around the crack tip and substituting their positions and stresses into Eq. (3), a linear set of simultaneous equations is obtained. By considering the stresses of $k$ nodes near the crack tip, the set of equations can be expanded in the following matrix form:

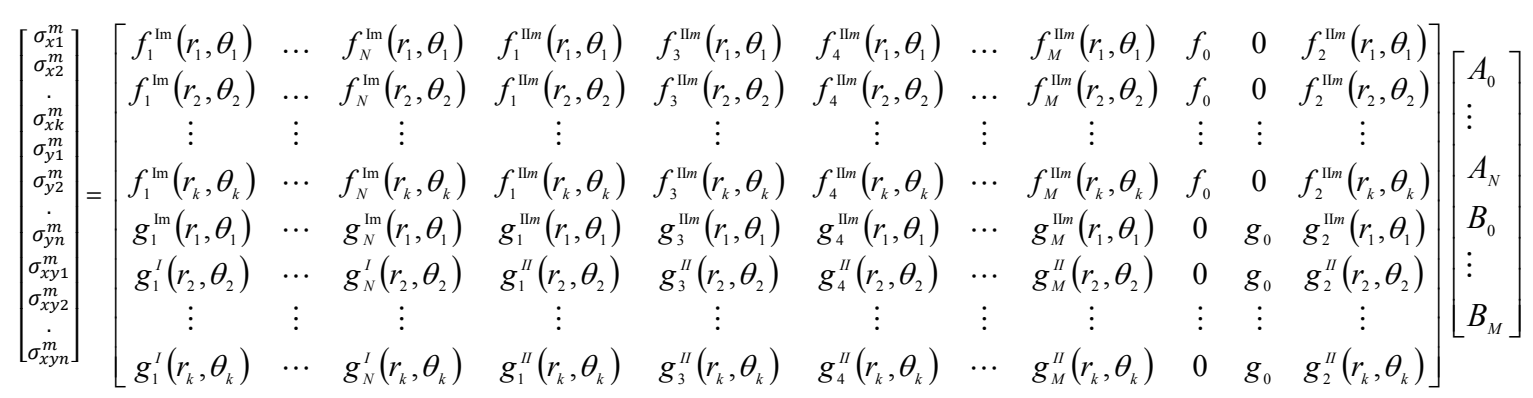

The terms containing $A_{0}$ to $A_{N}$ represent the mode $I$ part of stresses and similarly $B_{0}$ to $B_{\mathrm{N}}$, correspond to the mode $I I$ part of stresses in the William's series expansion.

\section{Description of the biomaterial model and numerical analysis}

Fig. 2 shows the geometry and loading condition of the bi-material plate with dimensions of $20 \times 20 \times 1$ in $^{3}$ containing a center crack of length $2 a=2$ in at the interface. The plate is loaded biaxialy by far field stress $\left(\sigma_{0}, \sigma_{1}, \sigma_{2}\right)$. Different material properties $\left(E_{1}, E_{2}\right.$ and $\left.v_{1}, v_{2}\right)$ were also considered for materials 1 and 2 to investigate the effects of material types on the interface fracture parameters. Table 1 summarize different loading and material cases considered for analyzing the bi-material center crack of this research.

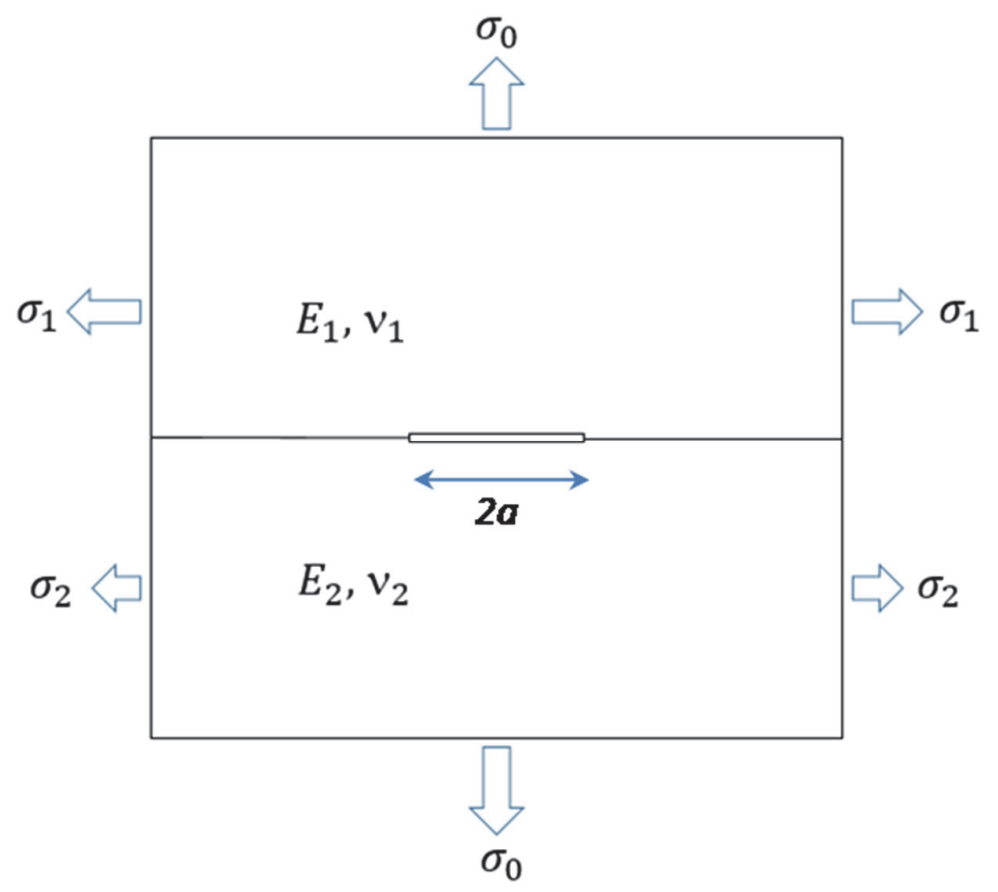

Fig. 2. Geometry and loading condition of a bi-material plate containing a center crack and subjected to general biaxial loading 
Table 1. Material properties and loading conditions of the investigated bi-material center cracked plate

\begin{tabular}{cccccccc}
\hline Case. No & $E_{1}(\mathrm{psi})$ & $E_{2}(\mathrm{psi})$ & $v_{1}$ & $v_{2}$ & $\sigma_{0}(\mathrm{psi})$ & $\sigma_{1}(\mathrm{psi})$ & $\sigma_{2}(\mathrm{psi})$ \\
\hline 1 & 1.0 & 1.0 & 0.3 & 0.3 & 1.0 & 0.0 & 0.0 \\
2 & 1.0 & 0.3333 & 0.3 & 0.3 & 1.0 & 1.0 & 0.53 \\
3 & 1.0 & 0.1 & 0.3 & 0.3 & 1.0 & 1.0 & 0.37 \\
4 & 1.0 & 0.045 & 0.3 & 0.35 & 1.0 & 1.0 & 0.38 \\
5 & 1.0 & 0.01 & 0.3 & 0.3 & 1.0 & 1.0 & 0.31 \\
6 & 1.0 & 0.0072 & 0.3 & 0.35 & 1.0 & 1.0 & 0.36 \\
7 & 1.0 & 0.001 & 0.3 & 0.3 & 1.0 & 1.0 & 0.3 \\
\hline
\end{tabular}

In order to compute three fracture parameters for the investigated interface fracture problem, it is necessary to determine the elastic stress field in the vicinity of crack tip. Based on the previous section, the nodal stress field obtained from the finite element analysis of biaxial center crack can then be inserted into the FEOD method (i.e. Eq. 4) to determine the stress intensity factors $\left(K_{\mathrm{I}}\right.$ and $\left.K_{\mathrm{II}}\right)$ as well as the $T$-stress. Hence first the finite model of the center cracked plate was created using eight node plane strain elements (as shown in Fig. 3) and then a simple static FE analysis were performed for any of the cases given in Table 1 .

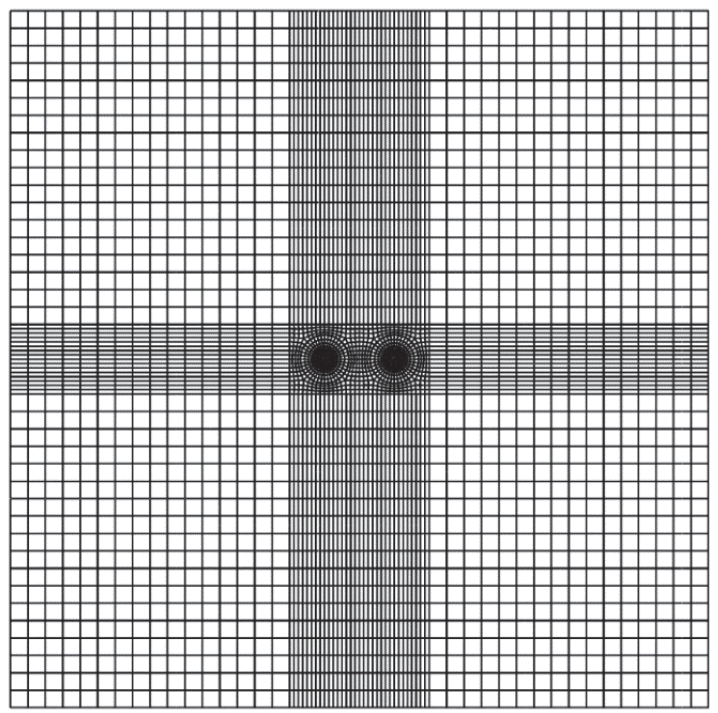

Fig. 3. Finite element model of center cracked bi-material plate

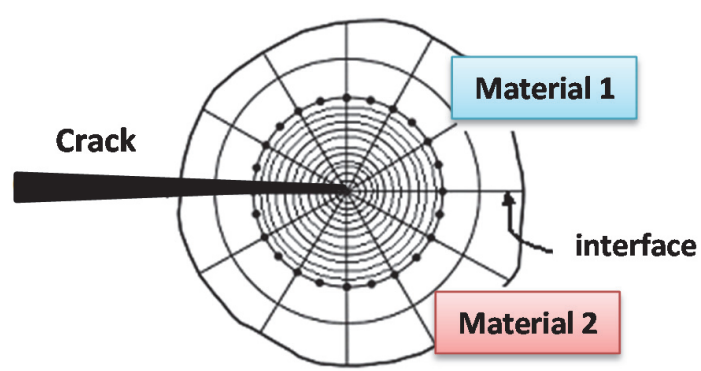

Fig. 4. Nodal stresses considred along a ring in both materials 1 and 2 for using in FEOD method

Next, as shown schematically in Fig. 4 the stress components (i.e. $\sigma_{r r}, \sigma_{r \theta}, \sigma_{\theta \theta}$ ) were obtained for the entire nodes of ring with radius of $r$ (surrounding the crack tip ) as proposed in Ayatollahi and Nejati (2011).

For proper convergence of results $r$ should be taken in the range of $\frac{a}{4}<r<\frac{a}{2}$, hence we considered typical value of $r=0.3$ in for extracting the nodal stress values. The nodal stresses were then replaced into Eq. 4, for FEOD calculation and the corresponding values of $K_{\mathrm{I}}, K_{\mathrm{II}}$ and $T$ (i.e. components of matrix $[\mathrm{X}]$ ) were determined for each case of plate model described in Table 1 . The nodal stresses of upper and lower rings were also used for materials 1 and 2, respectively in Eq. 4 . In the next section the obtained fracture parameters are presented and discussed.

\section{Results \& discussion}

Fig. 5 shows the $K_{\mathrm{I}}, K_{\mathrm{II}}$ and $T$ values for the analyzed bi-material interface center cracked plates subjected to different biaxial loads. Since materials 1 and 2 are identical in case 1 (see Table 1), this loading case represents the conventional homogenous center cracked plate under far field tension, in 
which a closed form solution is available for determining its fracture parameter. Based on the pioneer work of Williams and Ewing (1972), fracture parameters of the center cracked plate under pure mode I is determined from $K_{\mathrm{I}}=\sigma_{0} \sqrt{\pi a}, K_{\mathrm{II}}=0$ and $T=-\sigma_{0}$. The numerical results of $K_{\mathrm{I}}, K_{\mathrm{II}}$ and $T$, computed from the FEOD method is exactly identical with the analytical solution which can be considered as the validity of the numerical stress based FEOD calculations. The highest value of $K_{\mathrm{I}}$ and $T$ is obtained when the plate is homogenous. But by increasing the bi-material constant $(\varepsilon), K_{\mathrm{I}}$ and $T$ decrease while $K_{\text {II }}$ becomes more.
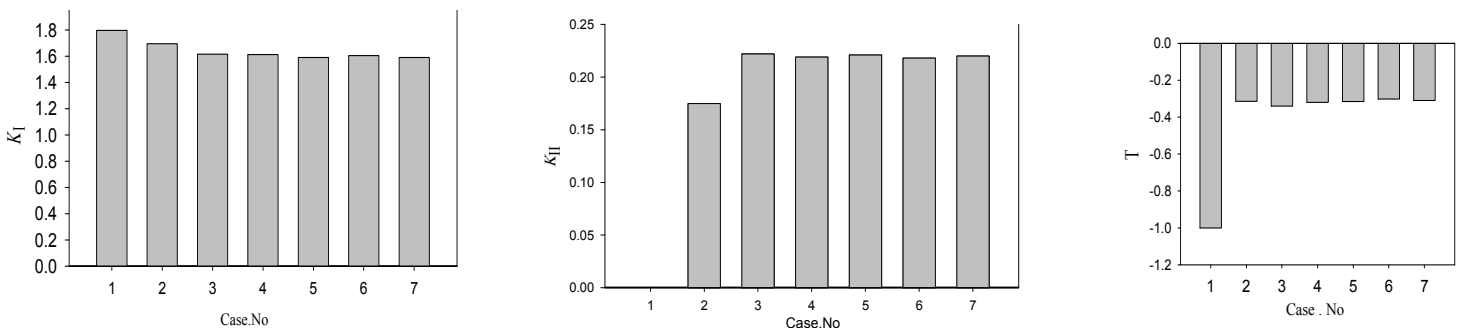

Fig. 5. Corresponding values of $K_{\mathrm{I}}, K_{\mathrm{II}}$ (in: psi.in ${ }^{0.5}$ ) and $T$ (in: psi) for different loading cases of investigated bi-material center cracked plate

The stress intensity amplitude $K_{0}$, defined by $K_{0}=\sqrt{K_{I}^{2}+K_{I I}^{2}}$ can be considered as a meaning full parameter in the analyzed plate, since it can be related directly to the energy release rate. Fig. 6 presents variations of $K_{0}$ for different center cracked plates investigated in this research.

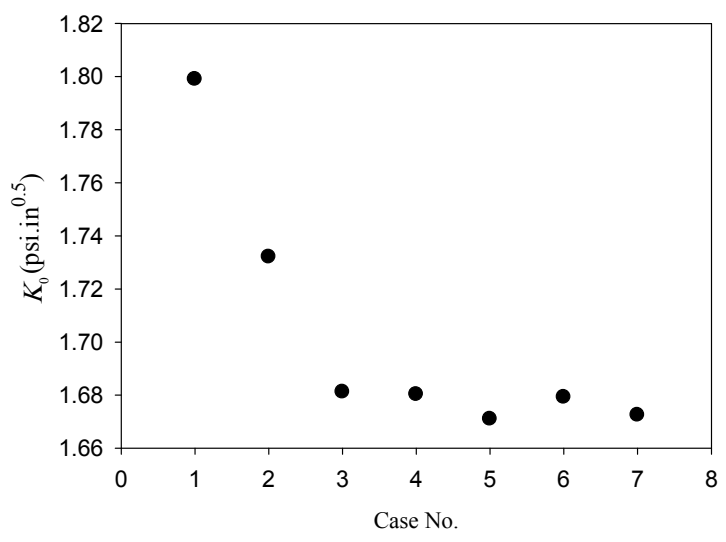

Fig. 6. Variations of stress intensity amplitude $\left(K_{0}\right)$ for different center cracked plates

Using the displacement field method, Chen (1985) determined the $K_{\mathrm{I}}$ and $K_{\mathrm{II}}$ values for the same bi-material center cracked plate. In order to compare the stress intensity factor results obtained from the stress and displacement fields, the $K_{0}$ values of these two methods are presented in Table 2. It is seen that a good consistency exists between the two sets of result such that the maximum discrepancy of two methods is less than $7 \%$. By increasing the bi-material constant, the magnitude of $T$-stress, decreases noticeably implying that unlike the homogonous center crack plate, the influence of nonsingular stress term are not significant for the case of bi-material problem. Furthermore, as stated earlier the case- 1 in Table 1 corresponds to pure mode $I$ loading condition because of symmetry in geometry, material and loading relative to the crack plane. However, the interface crack in other cases in Table 1 would experience mixed mode $I / I I$ loading condition due to asymmetry of material or loading relative to the interface line. Indeed, as seen from Fig. 7, while the crack tip stress contour is symmetric with respect to the interface line, the stress contour of case 4 (for instance) is not symmetric and crack experiences combined opening and sliding deformations. 
Table 2. Comparison of $K_{0}$ values determined from the stress and displacement field methods

\begin{tabular}{lccc}
\hline \multirow{2}{*}{ Case. NO } & \multicolumn{2}{c}{ Values of stress intensity amplitude $\left(\mathrm{K}_{0}\right)$} & \\
\cline { 2 - 3 } & $\begin{array}{c}\text { Present study (stress field) } \\
\left(\mathrm{psi.in}^{0.5}\right)\end{array}$ & $\begin{array}{c}\text { Chen }(\text { displacement field) } \\
\left(\mathrm{psi.in}^{0.5}\right)\end{array}$ & Error \% \\
\hline 1 & 1.7990 & 1.8090 & 0.5559 \\
2 & 1.7321 & 1.7980 & 3.8071 \\
3 & 1.6812 & 1.7830 & 6.0548 \\
4 & 1.6803 & 1.7820 & 6.0499 \\
5 & 1.6710 & 1.7710 & 5.9871 \\
6 & 1.6792 & 1.7790 & 5.9414 \\
7 & 1.6725 & 1.7690 & 5.7728 \\
\hline
\end{tabular}

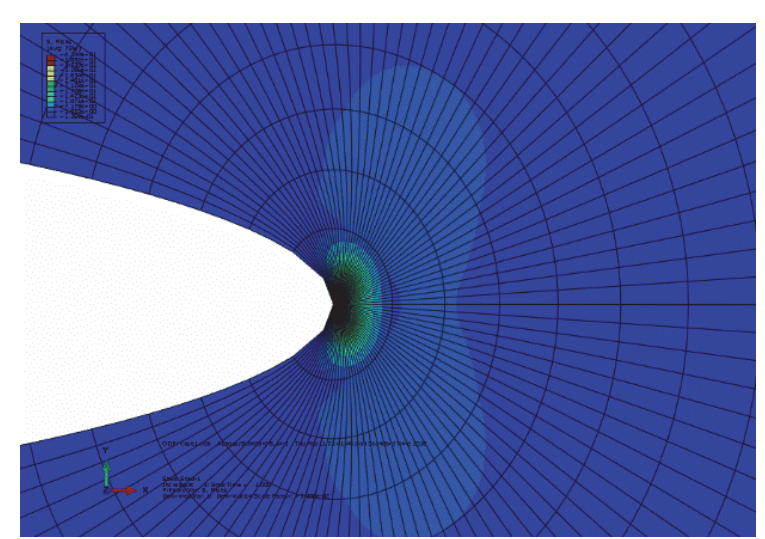

(a)

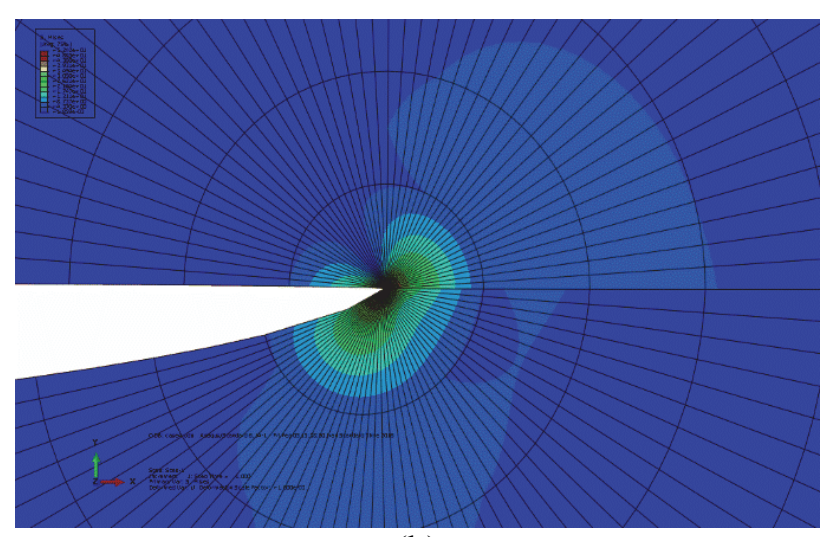

(b)

Fig. 7. Crack tip stress contours for (a) case "1" and (b) case "4" loading conditions

\section{Conclusions}

1. Three fracture parameters of biomaterial plate containing a center crack were computed numerically under different biaxial loading cases.

2. Using the crack tip stress field and by employing the finite element over deterministic (FEOD) method, $K_{\mathrm{I}}$ and $K_{\mathrm{II}}$ and $T$ stress values were computed.

3. The investigated bi-material cracked plate experiences a mixed mode opening-sliding fracture because of asymmetry in both material and loading relative to the interface line.

4. It was demonstrated that the fracture parameters of the bi-material center crack plate computed from the stress field method are accurate and very good agreement exists between the stress field and displacement field methods.

5. The amplitude of stress intensity factor $\left(K_{0}\right)$ which is related to the energy relase rate becomes smaller by increasing the bi-material constant value $\varepsilon$.

\section{References}

Abd-Elhady, A. (2013). Mixed mode I/II stress intensity factors through the thickness of disc type specimens. Engineering Solid Mechanics, 1(4), 119-128.

Aliha, M. R. M., Bahmani, A., \& Akhondi, S. (2015). Numerical analysis of a new mixed mode I/III fracture test specimen. Engineering Fracture Mechanics, 134, 95-110.

Aliha, M. R. M., Hosseinpour, G. R., \& Ayatollahi, M. R. (2013). Application of cracked triangular specimen subjected to three-point bending for investigating fracture behavior of rock materials. Rock mechanics and rock engineering, 46(5), 1023-1034.

Ayatollahi, M. R., Aliha, M. R. M., \& Saghafi, H. (2011). An improved semi-circular bend specimen for investigating mixed mode brittle fracture. Engineering Fracture Mechanics, 78(1), 110-123. 
Asaro, R. J., O'Dowd, N. P., \& Shih, C. F. (1993). Elastic-plastic analysis of cracks on bimaterial interfaces: interfaces with structure. Materials Science and Engineering: A, 162(1), 175-192.

Ayatollahi, M. R., \& Aliha, M. R. M. (2009). Analysis of a new specimen for mixed mode fracture tests on brittle materials. Engineering Fracture Mechanics, 76(11), 1563-1573.

Ayatollahi, M. R., \& Aliha, M. R. M. (2007). Wide range data for crack tip parameters in two disc-type specimens under mixed mode loading. Computational materials science, 38(4), 660-670.

Ayatollahi, M. R., \& Nejati, M. (2011). An over-deterministic method for calculation of coefficients of crack tip asymptotic field from finite element analysis. Fatigue \& Fracture of Engineering Materials \& Structures, 34(3), 159-176.

Chen, E. P. (1985). Finite element analysis of a bimaterial interface crack. Theoretical and Applied Fracture Mechanics, 3(3), 257-262.

Ensaff, H., O'Doherty, D. M., \& Jacobsen, P. H. (2001). The influence of the restoration-tooth interface in light cured composite restorations: a finite element analysis. Biomaterials, 22(23), 3097-3103.

Hakimzadeh, S., Kebede, N. A., Buttlar, W. G., Ahmed, S., \& Exline, M. (2012). Development of fracture-energy based interface bond test for asphalt concrete. Road materials and pavement design, 13(sup1), 76-87.

Hutchinson, J. W., \& Suo, Z. (1992). Mixed mode cracking in layered materials. Advances in applied mechanics, 29(63), 191.

Mirsayar, M. M., \& Park, P. (2016). Modified maximum tangential stress criterion for fracture behavior of zirconia/veneer interfaces. Journal of the Mechanical Behavior of Biomedical Materials, 59, 236240.

Mirsayar, M., \& Samaei, A. (2014). Application of maximum tangential stress criterion in determination of fracture initiation angles of silicon/glass anodic bonds. Engineering Solid Mechanics, 2(3), 145-150.

Mirsayar, M. (2013). Calculation of stress intensity factors for an interfacial notch of a bi-material joint using photoelasticity. Engineering Solid Mechanics, 1(4), 149-153.

Mirsayar, M. M., Aliha, M. R. M., \& Samaei, A. T. (2014). On fracture initiation angle near bi-material notches-Effects of first non-singular stress term. Engineering Fracture Mechanics, 119, 124-131.

Mirsayar, M. M. (2014). On fracture of kinked interface cracks-The role of T-stress. Materials \& Design, 61, 117-123.

Morioka, Y., \& Sun, C. T. (2010, April). A Simple Method for Determining Stress Intensity Factors in Bi-material Interfacial Cracks. In 51st AIAA/ASME/ASCE/AHS/ASC Structures, Structural Dynamics, and Materials Conference 18th AIAA/ASME/AHS Adaptive Structures Conference 12th (p. 2561).

Ravichandran, M., \& Ramesh, K. (2005). Evaluation of stress field parameters for an interface crack in a bimaterial by digital photoelasticity. The Journal of Strain Analysis for Engineering Design, 40(4), 327-344.

Rice, J. R., \& Sih, G. C. (1965). Plane problems of cracks in dissimilar media. Journal of Applied Mechanics, 32(2), 418-423.

Toparli, M., \& Aksoy, T. (1998). Fracture toughness determination of composite resin and dentin/composite resin adhesive interfaces by laboratory testing and finite element models. Dental Materials, 14(4), 287-293.

Wang, X., \& Agrawal, C. (2000). A mixed mode fracture toughness test of bone-biomaterial interfaces. Journal of biomedical materials research, 53(6), 664-672.

Williams, M. L. (1959). The stresses around a fault or crack in dissimilar media. Bulletin of the seismological society of America, 49(2), 199-204.

Williams, J. G., \& Ewing, P. D. (1972). Fracture under complex stress - the angled crack problem. International Journal of Fracture Mechanics, 8(4), 441-446.

Zhong, H., Ooi, E. T., Song, C., Ding, T., Lin, G., \& Li, H. (2014). Experimental and numerical study of the dependency of interface fracture in concrete-rock specimens on mode mixity. Engineering Fracture Mechanics, 124, 287-309. 